\title{
Development of LMS Algorithm with Adapted and Unadapted Step Size
}

\author{
Mohaned Mohammed Hani, Asmaa Hameed Rasheed \\ University of Baghdad \\ Iraq \\ mohaned.m.h2013@gmail.com \\ d.asmaamoaath@gmail.com
}

\begin{abstract}
Adaptive algorithms play a major role in all adaptive processing systems. One of the most important and wellknown algorithms is the least mean square (LMS) algorithm due to simplicity, stability and fast convergence rate. In this paper, two different types of the LMS algorithm were reviewed and analyzed: adapted and unadapted variable step size. The adapted step size varies its value according to signal features while unadapted step size varies its value with a fixed predefined constant. The performance of each algorithm measured in the scope of adaptive noise canceling system. Performance quality was determined with respect to mean square error (MSE), convergence rate and algorithm stability. According to the obtained simulation results the Adapted variable step size VSS-LMS algorithm was performed very well and converged rapidly also it was stable through the adaptation process, as a result, it behaves better than unadapted VSS-LMS algorithm. And both adapted and unadapted VSS-LMS algorithms give an enhanced performance for the ordinary fixed step size LMS algorithm.
\end{abstract}

Keywords: LMS Algorithm, Adaptive Algorithms, Variable Step Size (VSS), Adaptive Noise Cancelling (ANC)

DOI: $10.6025 /$ jitr/2018/9/3/85-93

Received: 1 April 2018, Revised 5 May 2018, Accepted 14 May 2018

(C) 2018 DLINE. All Rights Reserved

\section{Introduction}

In the applications which requires adaptation, the least mean square (LMS) algorithm is one of the most frequently used algorithms. It has a lot of great characteristics that made it one of the well-known adaptation algorithm, It is simple, has a fast convergence time and it is robustly stable. One of the applications where the LMS algorithm is usually used is adaptive noise canceling. Although the LMS algorithm is simple, it is a complex operation to do the complete mathematical analysis of the algorithm and defining the exact rules for the step size adjustment probably because of the nonlinear character of the algorithm and the non-stationary behavior of noise. The variable step size VSS-LMS was devised as a solution to make the adaptation as fast as possible by making the change in step size suitable to the application while keeping the performance robust and stable. For that purpose, every few years a new VSS-LMS appears with the goal of being practically useful in the applications where it is used. An illustration of the operation of the LMS algorithm is shown in Figure 1 [1]. 


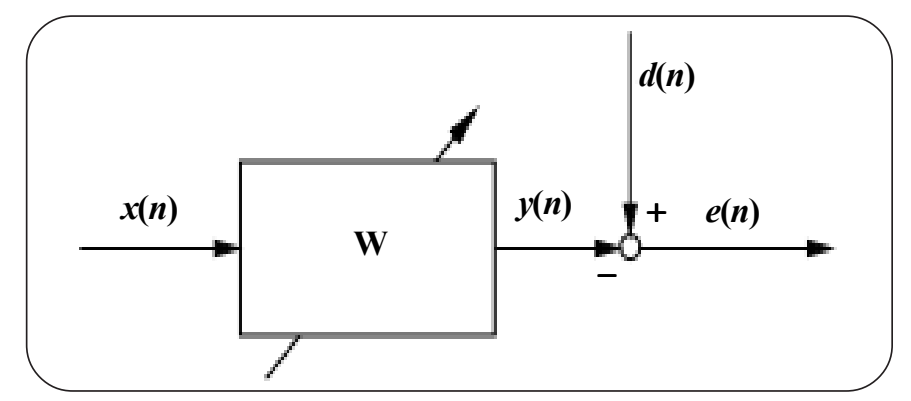

Figure 1. Adaptive filtering problem

The input to the adaptive filter $W$ is the sequence $x(n)$. An error signal $e(n)$ is generated by subtracting $y(n)$ which is the filter output from the desired signal $d(n)$. The adaptive algorithm changes the filter coefficients by adjusting them to minimize the error. The adaptive filter is considered to be of finite impulse response (FIR) type with the taps stored in row vector:

$$
w(n)=\left[w_{0}(n) w_{1}(n) \ldots w_{L-1}(n)\right]^{T}
$$

Here $T$ represents transpose, the filter taps are updated by the LMS algorithm according to the famous formula:

$$
w(n+1)=w(n)+\mu x(n) e(n)
$$

where $\mu$ represents the step size and $x(n)$ the row vector which contains the input signal's samples arranged regressively[2]:

$$
x(n)=\left[x(n) x(n-1) x(n-2) \ldots x(n-L+1)^{T}\right.
$$

The main constraints that limit the choice of the step size can be summarized as follows [3]:

Large step size leads to very fast convergence but make the MSE excess the allowed criteria and produce a slightly large MSE. Choosing too large step size may lead the algorithm to diverge and as result of an unstable system which uses the LMS algorithm. On the other hand, too small step size slows down the convergence and results in a small value of MSE but despite of that some applications require fast response of the algorithm, so too small values of step size may be impractical especially in the applications of adaptive noise canceling. At this point, the importance of the VSS-LMS becomes clear because it achieves the trade-off between speed of convergence, the desired steady-state of misadjustment, and the stability of the system.

\section{Adaptive Algorithms}

Several variable step size LMS algorithm had emerged in the past few years with the aim of improving the speed of convergence and the tracking ability of the signal contaminated by noise.

These modified LMS algorithms provide very well performance compared to the ordinary LMS algorithm. In this paper, we did an analysis of three types of the LMS algorithms which they are the standard LMS and constantly variable step size LMS and VSS-LMS and these algorithms were tested in real time noisy environment for adaptively denoising speech signal.

\section{The LMS Algorithm}

One of the simplest and most frequently used algorithms in the adaptive filters is LMS algorithm because of its ease of implementation and low computational complexity. Filter weights update of the adaptive noise canceller and the error equations are given by:

$$
\begin{aligned}
& w(n+1)=w(n)+\mu x(n) e(n) \\
& e(n)=d(n)-w^{T}(n) x(n)
\end{aligned}
$$


Where $n$ is the time index, bolded symbols represent vectors, $w(n)$ is an $L \times 1$ vector of the adaptive filter coefficients, $L$ is the adaptive filter length, is the step size which controls the convergence and the steady-state misadjustment and by means the performance of the LMS algorithm. An approximation of the convergence condition is [4]:

$$
0.00001<\mu<\frac{2}{L S_{\max }}
$$

The major disadvantage of the LMS based adaptive noise canceller is that the steady-state misadjustment linearly increases with the desired signal power. This leads to high degradation in the performance especially when the desired signal exhibits large power fluctuations and this is a major drawback for the LMS in the field of speech processing[5].

\section{The Unadapted LMS Algorithm}

In this section a proposed modification to the LMS algorithm is done by introducing a constant $C$ on the step size and making the sign of that constant alternates between positive and negative depending on the measured mean square error so if the mean square error is decreased in the $n^{\text {th }}$ iteration the step size is increased by the value of $C$ in the $(n+1)^{\text {th }}$ and vise-versa. The filter update equation and the convergence criteria is given by:

$$
\begin{gathered}
w(n+1)=w(n)+\mu x(n) e(n) \\
\mu(n+1)=\mu(n)+C \\
C=\left\{\begin{array}{l}
+C \text { if the } \operatorname{MSE}(n)<\operatorname{MSE}(n-1) \\
-C \text { if the } \operatorname{MSE}(n)>\operatorname{MSE}(n-1) \\
+C \text { if the } \operatorname{MSE}(n)=\operatorname{MSE}(n-1)
\end{array}\right. \\
e(n)=d(n)-w^{T}(n) x(n)
\end{gathered}
$$

The value of $C$ is chosen according to the application where the algorithm used, in our case $C$ was chosen to equal 0.001 for convenience. The main advantage of this algorithm is that the step size will vary until finding the minimum value of the MSE but it also has a major disadvantage of being slightly slow to converge.

\section{The Adapted Vss-Ims Algorithm By Kwong et al.}

In this section, another proposed modification to the LMS algorithm which relates the error directly to the step size is proposed by Kwong et al.[6] is given by:

$$
\begin{gathered}
\mu^{\prime}(n+1)=\alpha \mu(n)+\gamma e^{2}(n) \\
\mu^{\prime}(n+1)= \begin{cases}\mu_{\max } & \text { if } \mu^{\prime}(n+1)>\mu_{\max } ; \\
\mu_{\min } & \text { if } \mu^{\prime}(n+1)<\mu_{\min } ; \\
\mu^{\prime} & \text { Otherwise; }\end{cases}
\end{gathered}
$$

Where $0<\alpha<1$ and $\gamma>0$ are a tuning parameters, and $0<\mu_{\min }<\mu_{\max }$ are selected to provide a good tracking ability and stability respectively. The value of $\alpha$ was selected to equal to 0.92 . The parameter $\gamma$ affects the speed of convergence and the MSE so it should be chosen carefully and with a very small value which was in our case equal to 0.00042 . The parameter $\mu_{\max }$ was chosen to be 0.6 for practical considerations. The developers of this algorithm have developed it in such a way where the step size is directly dependent on the square error. The main idea is when the error is too large a fast adaptation is required, so the step size is to be made large then the step size to be decreased when the error is degraded.

\section{Simulation Results}

Journal of Information Technology Review Volume 9 Number 3 August 2018 
In this section, the obtained results are demonstrated. The performance of each algorithm is analyzed individually based on the parameters that are mentioned earlier which they are the mean square error, the speed of convergence and stability of the

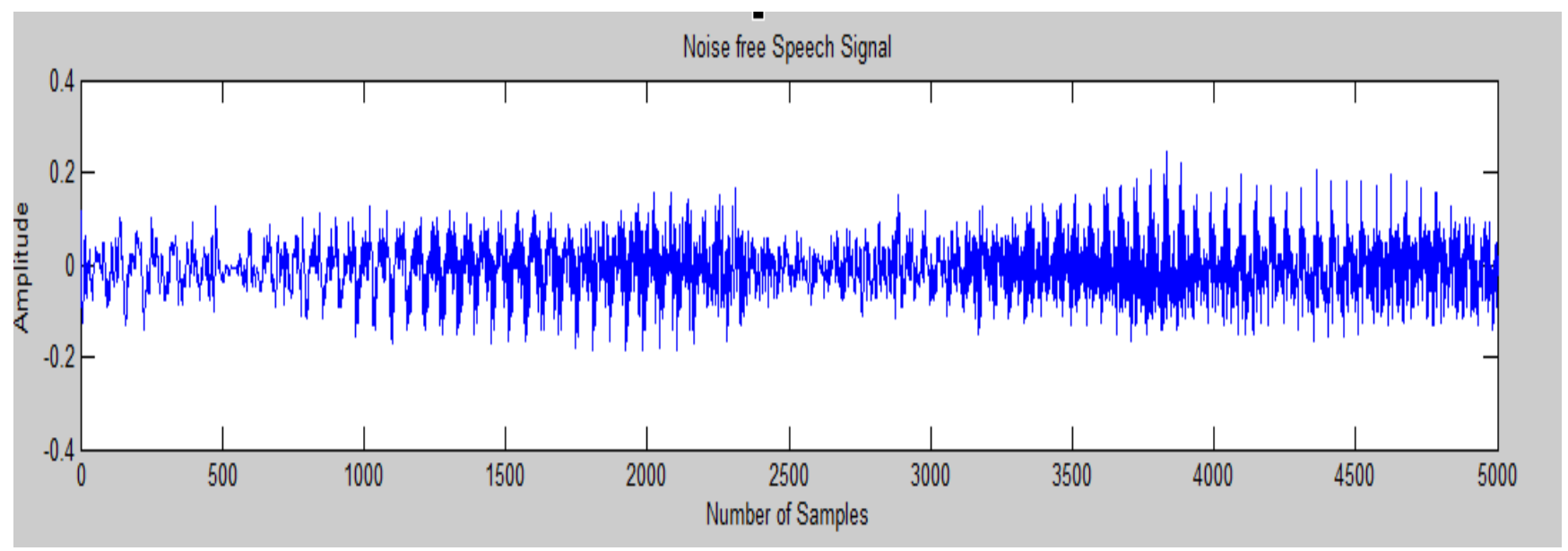

Figure 2. Noise-free speech signal

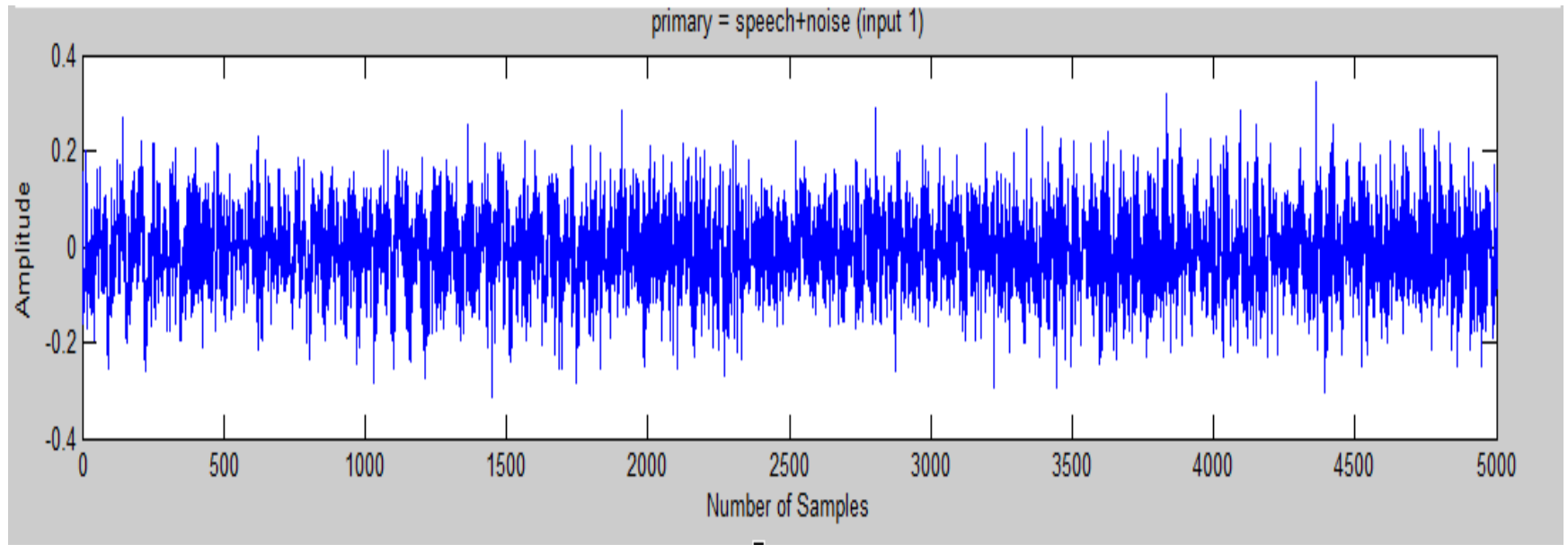

Figure 3. Noise signal

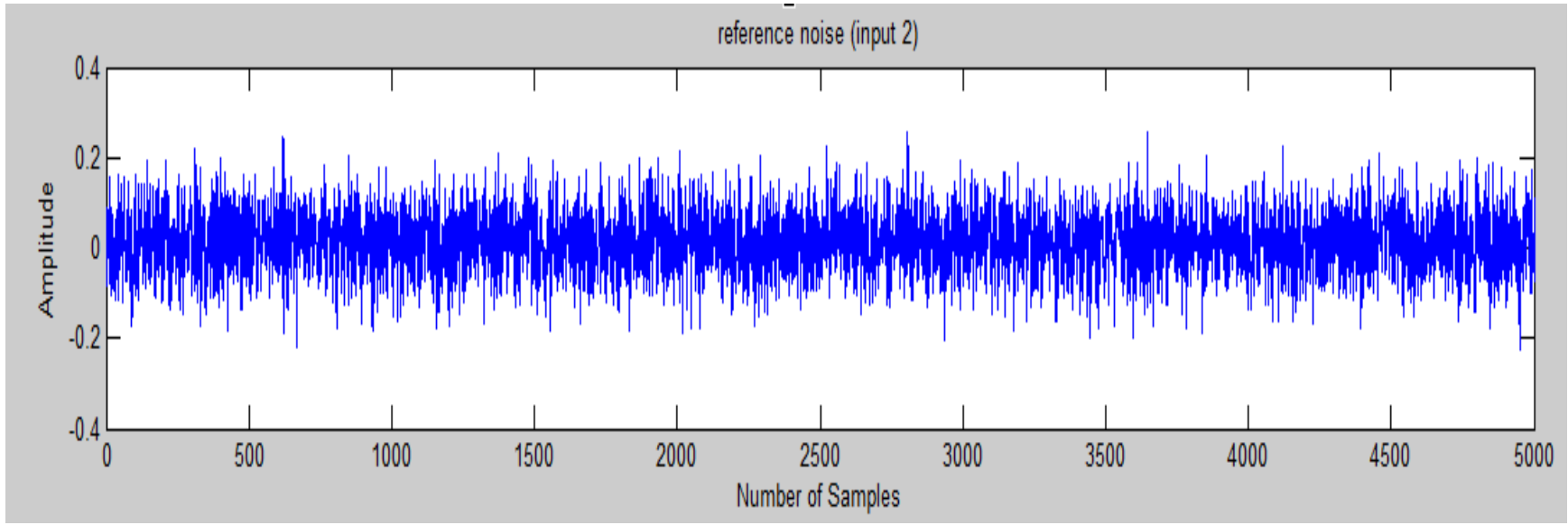

Figure 4 . The noisy signal at the input of the adaptive filter 
algorithm while varying one or more algorithm parameter, in our case varying the step size. This paper assumed that the algorithms operate in an actual noisy environment and the simulation was done based on that assumption. The adaptive filter is chosen of the finite impulse response (FIR) with a number of taps equal to 10. A speech signal with a sampling frequency of $44100 \mathrm{KHz}$ is used in the simulation and shown in figure 2 was assumed to be contaminated by white noise as shown in figure 3. The resultant noisy signal (speech + noise) is shown in figure 4 .

The simulation results showed that the standard LMS algorithm adapted to reduce the noise but not in a perfect manner so the signal which is obtained at the adaptive filter output is quite noisy as shown in figure 5.

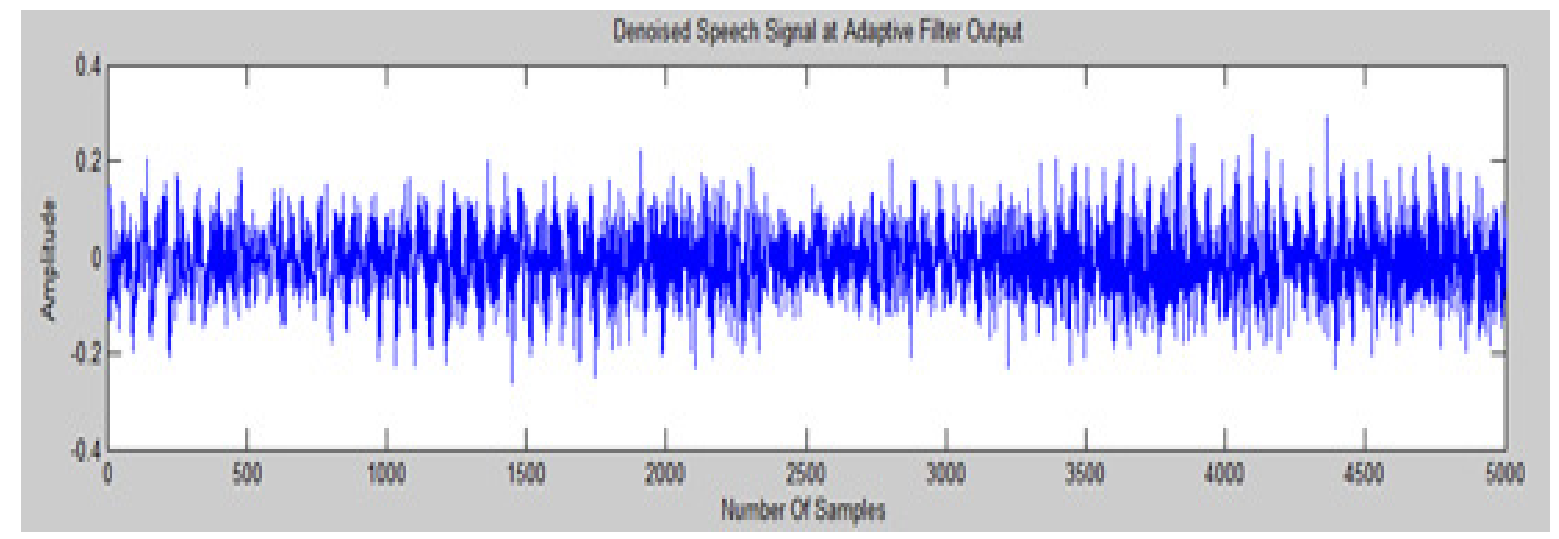

Figure 5. LMS adaptive filter denoised output signal

The step size chosen in the simulation was equal to 0.001. A review of the performance of the standard LMS algorithm can be illustrated by the MSE vs. the number of iteration curve shown in figure 6.

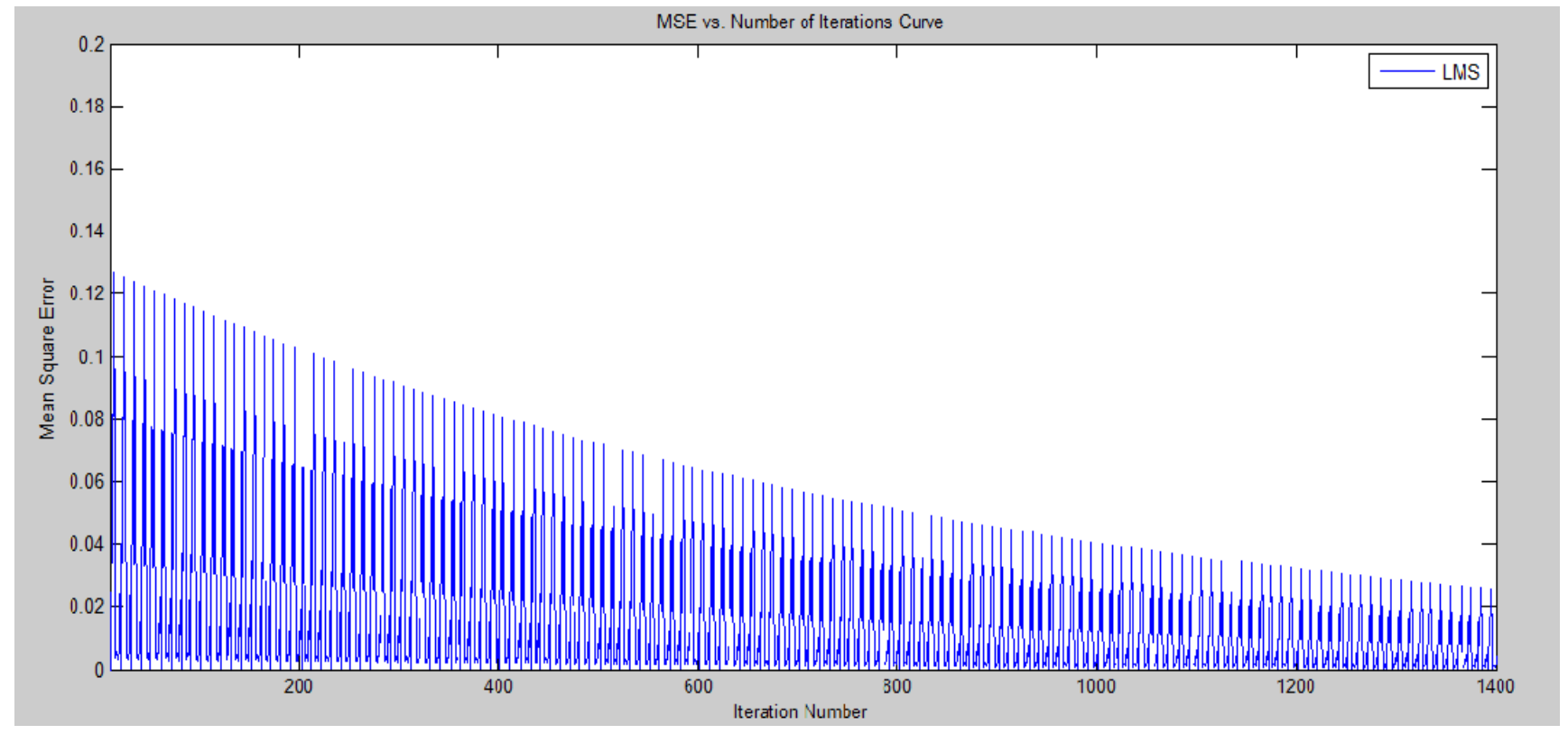

Figure 6. LMS MSE vs. the number of iterations curve

The final value of the mean square error estimated from fig. 6 was about 0.023 which reveals about $18 \%$ error or misadjustment by the algorithm to reduce the noise. The learning curve is obtained by smoothing the MSE vs. number of iterations curve in figure 6 and is illustrated in figure 7. 


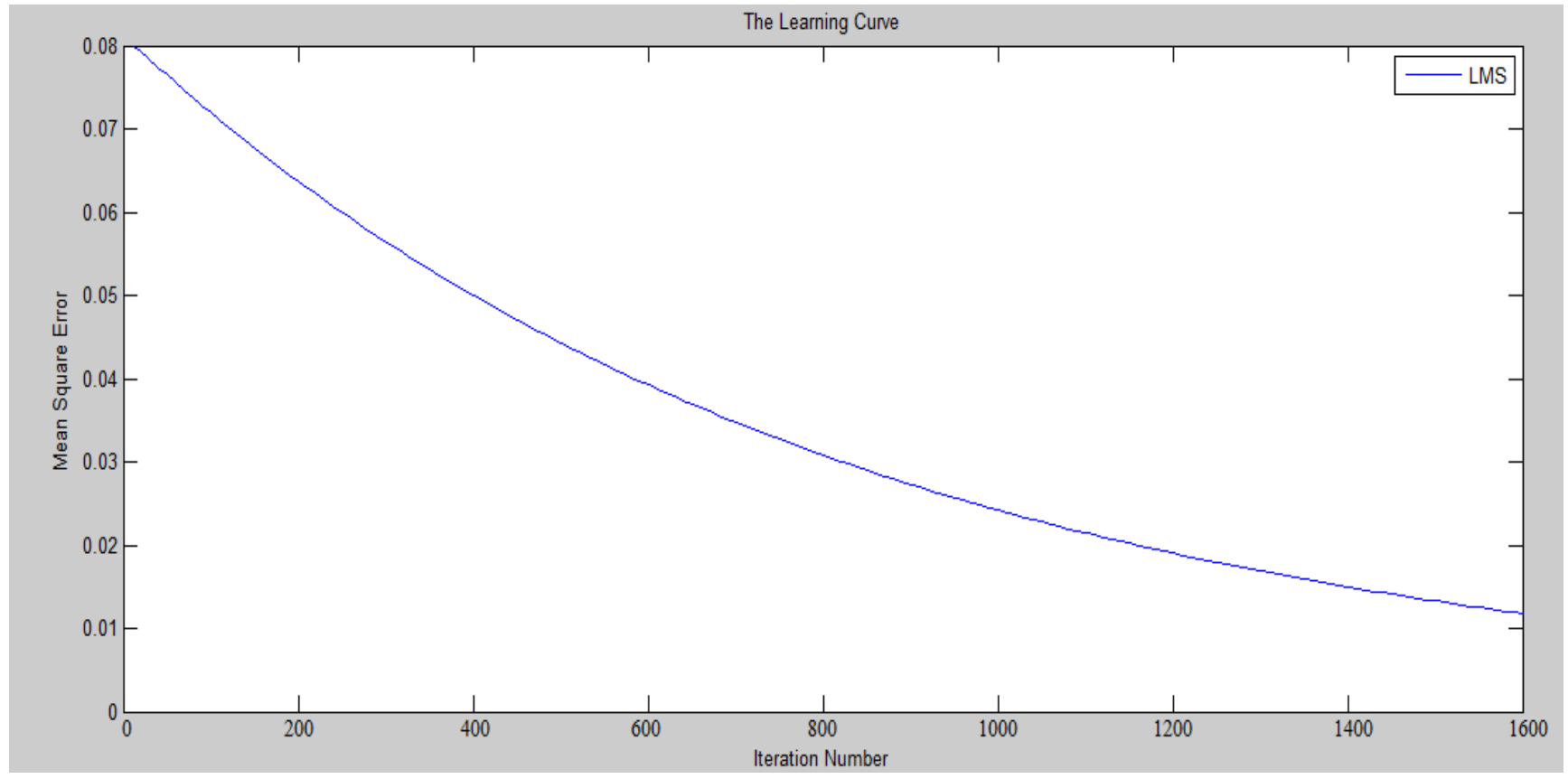

Figure 7. LMS algorithm's learning curve

Although the algorithm was stable it consumed a large time (larger number of iterations) to converge, the algorithm consumed about 1500 iterations to converge. When the FSS-VSS LMS was used the output of the filter is shown in figure 8 and the noise is more reduced.

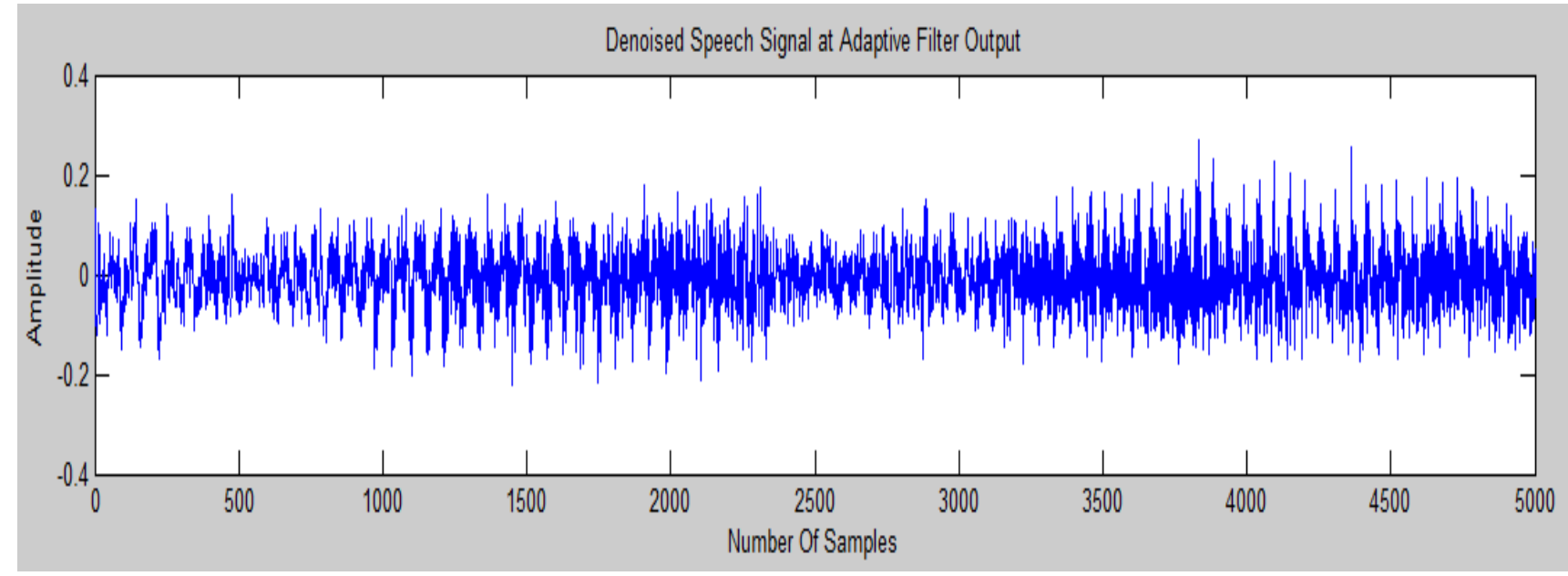

Figure 8. FSS-VSS LMS adaptive filter denoised output signal

The FSS-VSS LMS is performed slightly better than standard LMS as shown in fig. 9 where the final value of MSE is reduced to about 0.012 .

It is obvious that this algorithm is adapted well and was faster than LMS, so it consumed about 550 iterations to converge. The initial step size 0.001 and the constant $\mathrm{C}=0.0001$. The learning curve of the FSS-VSS LMS is shown in figure 10 . The bestadapted algorithm was the VSS-LMS, the adaptive filter was successfully extracted the desired signal from the noisy signal and the output was identical to the original signal by $99.9 \%$ as shown in figure 11 . The final MSE nearly vanishes and the misadjustment 
was very reduced nearly to zero. In Figure 12 an illustration the MSE vs. number of iteration curve.

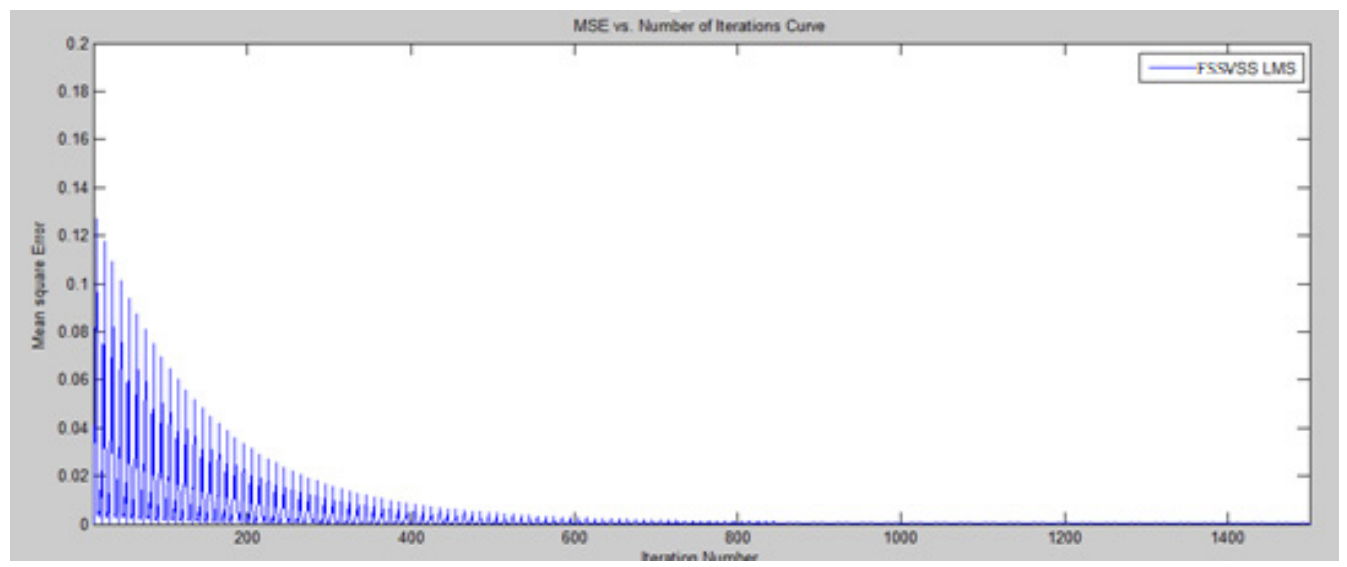

Figure 9. FSS-VSS LMS MSE vs. number of iterations curve

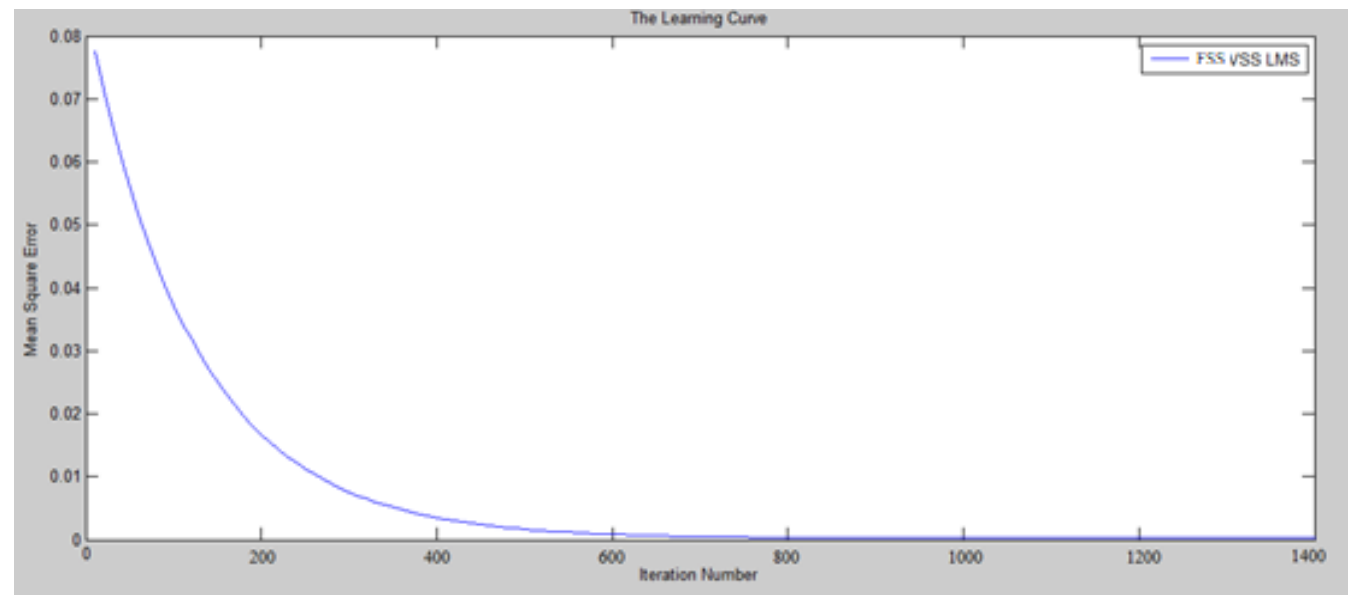

Figure 10. FSS-VSS LMS algorithm's learning curve

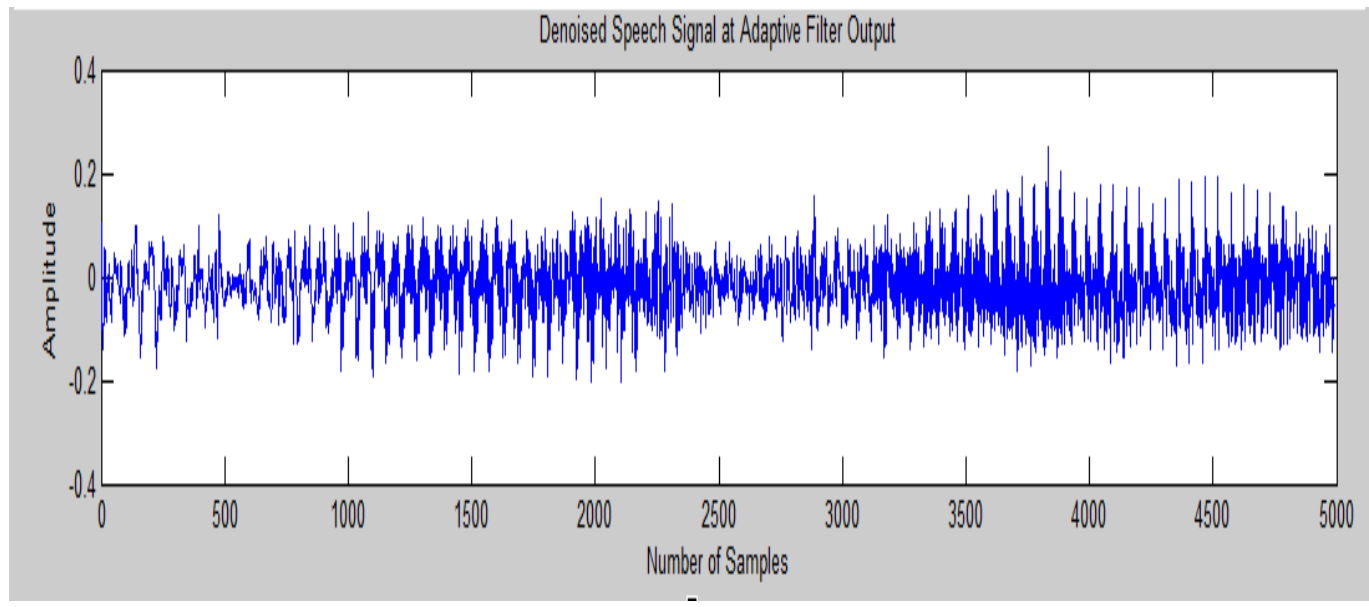

Figure 11. VSS-LMS adaptive filter denoised output signal 


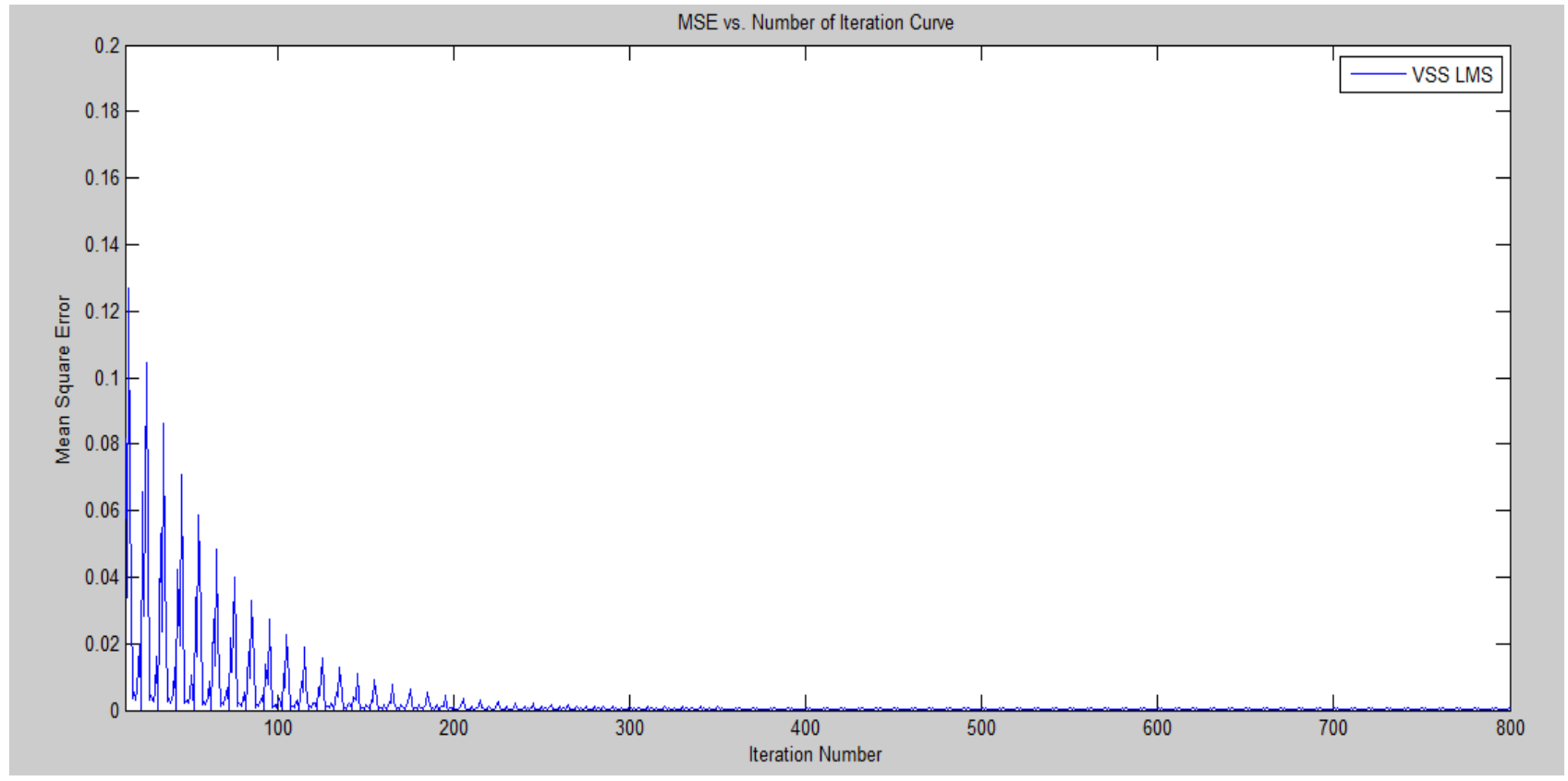

Figure 12. VSS-LMS MSE vs. number of iterations curve

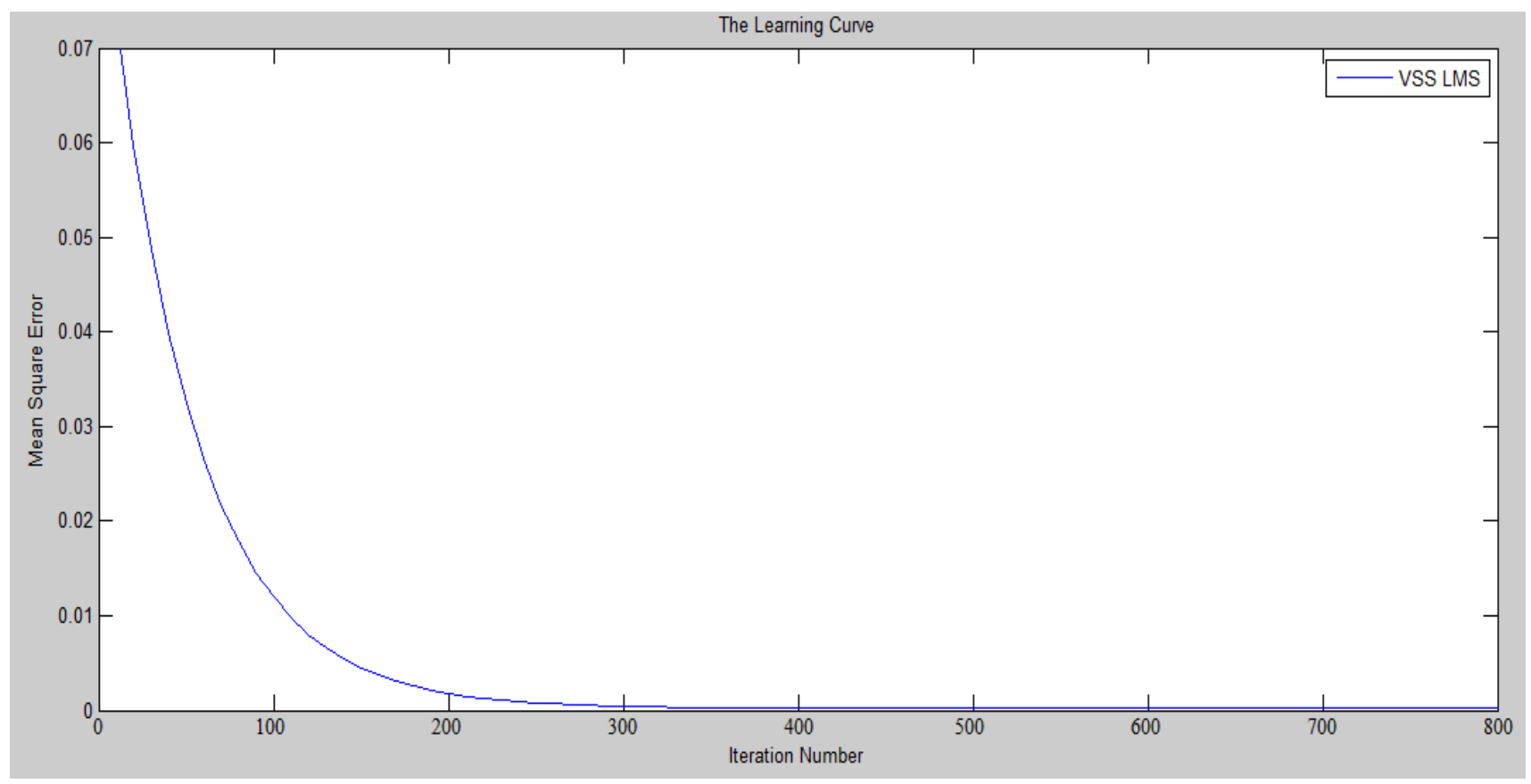

Figure 13. VSS-LMS algorithms learning curve

The performance of VSS-LMS can be judged from the learning curve which is shown in fig.13. The algorithm was converged rapidly and consumed less than 200 iterations also, it was adapted well and the MSE is reduced to zero nearly.

The results of the simulation can be arranged a table for purpose of comparing them and to judge which algorithm was better for our application, results are given in table 1 : 


\begin{tabular}{|l|c|c|c|}
\hline \multirow{2}{*}{ Algorithm } & \multicolumn{3}{|c|}{ Parameter } \\
\cline { 2 - 4 } & Convergence Speed & FinalMSE & Percent NoiseReduction \\
\hline LMS & 1000 iterations & $23 \%$ & $78 \%$ \\
\hline FSSLMS & 550 iterations & $11 \%$ & $86 \%$ \\
\hline VSSLMS & 220 iterations & $5 \%$ & $99 \%$ \\
\hline
\end{tabular}

Table 1. The obtained simulation results

We see that the VSS-LMS is very suitable for noise canceling applications due to the high adaptation ability available to the algorithm by the modification which was proposed in [6].

\section{Conclusion}

The main conclusion was made from the present work that the LMS Algorithm is always stable and has acceptable convergence rate with different applied approaches. By applying adapted and unadapted step size its obvious that the adapted VSS-LMS algorithm has higher convergence rate than the unadapted VSS-LMS algorithm as well as the final MSE is less than that obtained with the unadapted algorithm. Another important concluded remark is the VSS-LMS algorithm with both adapted and unadapted techniques is highly better than ordinary LMS algorithm, so its preferable for variable step size to be dependent for any adaptation process.

\section{Acknowledgment}

The authors of this paper present their thanks to Dr. Rabab Hameed for reviewing the English language grammars.Also, they present thanks to professor Nuha A. S. Alwan for her notes which helped to complete this paper.

\section{References}

[1] Haykin, S. (2002). Adaptive Filter Theory, Fourth Edition. Prentice Hall, New York.

[2] Widrow, B., Glover, R., McCool, M., Kaunitz, J., Williams, C., Hearn, R., Zeidler, J., Eugene Dong, Jr., Goodlin, R. C. (1975). Adaptive noise canceling: Principles and applications. Proceedings of the IEEE, 63 (12)1692-1716.

[3] Bismor, D., Czyz, K., Ogonowski, Z. (2016). Review and Comparison of Variable Step-Size LMS Algorithms, Int. Journal of acoustics and vibrations. 21 (1) 24-39.

[4] Zhang, Qi., Yao, Yuancheng., Qin, Mingwei. (2012).An Uncorrelated Variable Step-size LMS Adaptive Algorithm, Journal of Emerging Trends in Computing and Information Sciences, 3 (11) (November).

[5] Greenberg, J. E. (1998). Modified LMS algorithms for speech processing with an adaptive noise canceller, IEEE Trans. Speech audio Process. 6 (4) 338-358, (July).

[6] Kwong, R. H., Johnston, E. W. (1992). Variable step size LMS algorithm. IEEE Transactions on Signal Processing, 40 (7) $1633-1642$. 